\title{
Immunotherapy for extensive stage small cell lung cancer
}

\author{
Jose M. Pacheco \\ Division of Medical Oncology, Department of Internal Medicine, University of Colorado Cancer Center, Aurora Colorado, USA \\ Correspondence to: Jose M. Pacheco, MD. Assistant Professor, Thoracic Oncology and Developmental Therapeutics, 1665 Aurora Court, Room 5309, \\ Mail Stop F704, Aurora Colorado 80045, USA. Email: jose.m.pacheco@cuanschutz.edu.
}

\begin{abstract}
Small cell lung cancer (SCLC) is an aggressive malignancy. Until recently the standard of care for newly diagnosed patients with extensive-stage disease was chemotherapy consisting of etoposide plus a platinum (EP). The median overall survival (OS) was only about 10 months with this systemic therapy. Immune checkpoint inhibitors were first evaluated as second or subsequent line treatments in extensive stage disease and later in combination with EP in the first-line setting. Recently two randomized phase III trials have demonstrated statistically improved OS with addition of a programmed death ligand-1 (PD-L1) inhibitor to EP. As a result, the standard of care for newly diagnosed patients with extensive-stage SCLC has changed for the first time in decades. However, many patients do not derive benefit from the addition of a PD-L1 inhibitor to EP. In this review we discuss first-line trials of chemoimmunotherapy in extensive stage SCLC and summarize data on second and subsequent line treatment with immune checkpoint inhibitors in immunotherapy-naïve patients. Additionally, we discuss potential biomarkers that could be utilized to select for which patients derive benefit from addition of a PD-L1 inhibitor to EP and propose ways to improve on first-line chemoimmunotherapy.
\end{abstract}

Keywords: Small cell lung cancer (SCLC); immunotherapy; atezolizumab; durvalumab; programmed death-1 (PD-1)

Submitted Jan 03, 2020. Accepted for publication Jan 29, 2020.

doi: $10.21037 /$ jtd.2020.01.37

View this article at: http://dx.doi.org/10.21037/jtd.2020.01.37

\section{Introduction}

Until recently etoposide plus platinum (EP) was the standard of care in most parts of the World for extensive stage small-cell lung cancer (ES-SCLC). The median overall survival (OS) with EP was approximately 10 months despite high initial objective response rates (ORRs) of $50-70 \%(1-4)$. It was hypothesized that SCLC may preferentially benefit from immunotherapy due to a high tumor mutational burden (TMB) and the presence of autoimmune paraneoplastic syndromes in some patients $(5,6)$. Because of this chemoimmunotherapy was evaluated as first-line therapy for ES-SCLC and recently two regimens have demonstrated superiority over $\mathrm{EP}(7,8)$.

This review focuses on immune checkpoint inhibitors with chemotherapy for newly diagnosed patients with ES-SCLC, with particular attention being paid to drugs inhibiting either cytotoxic T-lymphocyte antigen 4
(CTLA-4) or programmed death-1 (PD-1)/programmed death ligand-1 (PD-L1). Additionally, data on second and subsequent line immune checkpoint inhibitors for patients with ES-SCLC will be summarized. New strategies will be proposed to improve on first-line chemoimmunotherapy combinations. While biomarkers will be touched upon, they will be discussed more intensively in other parts of this focused issue.

\section{Methods}

Trials were identified by searching PubMed without date limits, abstracts from major medical society meetings since 2015 (American Association for Cancer Research Annual Meeting, American Society of Clinical Oncology Annual Meeting, European Society of Medical Oncology Annual Meeting and International Association for the Study of Lung Cancer World Conference on Lung Cancer), and 
clinicaltrials.gov using the keywords: atezolizumab and small cell, durvalumab and small cell, ipilimumab and small cell, nivolumab and small cell, pembrolizumab and small cell, tremelimumab and small cell, Caspian and durvalumab, and IMpower133 and atezolizumab. Trials were excluded if they did not evaluate immune checkpoint inhibitors or did not report efficacy data specific to ES-SCLC.

\section{Chemoimmunotherapy as first-line treatment for ES-SCLC}

\section{Ipilimumab plus chemotherapy}

\section{Ipilimumab plus carboplatin plus paclitaxel}

A randomized, double-blind, phase II, placebo-controlled trial evaluated ipilimumab plus carboplatin plus paclitaxel vs. carboplatin plus paclitaxel in ES-SCLC. The three treatment arms included carboplatin plus paclitaxel for up to 6 cycles with ipilimumab added from start of cycle 3 , carboplatin plus paclitaxel for up to 6 cycle with ipilimumab added from start of cycle 1 and carboplatin plus paclitaxel plus placebo for up to 6 cycles. There was a non-statistically improved OS with the addition of ipilimumab starting from cycle 3 of induction chemo, median OS was 12.9 months $(\mathrm{n}=42)$ vs. 9.9 months $(\mathrm{n}=45)$, HR 0.75 (95\% CI, 0.46-1.23), $\mathrm{P}=0.13$ (9). This resulted in ipilimumab being evaluated with EP in a subsequent phase III study.

\section{Ipilimumab plus etoposide plus platinum}

A randomized, phase III, placebo-controlled, double-blind trial evaluated EP with or without ipilimumab in newly diagnosed patients with ES-SCLC. The first 6 cycles of systemic therapy were administered every 21 days. EP was administered for cycles 1 and 2, it was continued in conjunction with ipilimumab or placebo during cycles 3 and 4. Ipilimumab or placebo were administered as monotherapies during cycles 5 and 6 . The platinum agent could be cisplatin or carboplatin. Cisplatin was administered at $75 \mathrm{mg} / \mathrm{m}^{2}$ on day 1 and carboplatin was dosed at an area under the concentration time curve (AUC) of 5 on day 1 . Etoposide was given at $100 \mathrm{mg} / \mathrm{m}^{2}$ on days $1-3$ of each cycle. During cycles 3 through 6, ipilimumab or placebo were administered at $10 \mathrm{mg} / \mathrm{kg}$ every 3 weeks on day 1 . From cycle 7 onwards ipilimumab or placebo were given at the same dose every 12 weeks. Ipilimumab or placebo were continued for up to 36 months of treatment (10).

The primary endpoint was OS in patients receiving at least one dose of ipilimumab or placebo. Patients with asymptomatic, untreated brain metastases could enroll, but those with a history of autoimmune paraneoplastic syndromes were excluded. Consolidative chest radiotherapy was not permitted in cases where there was measurable intrathoracic disease at start of therapy. However, prior chest radiation as part of chemoradiation for limited stage disease was allowed. Patients with a complete response (CR) or partial response (PR) to the first 6 cycles of therapy could receive optimal prophylactic cranial radiation (PCI). There were $11 \%$ of patients who received PCI on the EP plus ipilimumab arm and $14 \%$ who received PCI on the EP alone arm (10).

The best ORR by investigator assessment was $62 \%$ on each arm and median duration of response (DOR) was similar at $4 v s .3 .5$ months with the addition of ipilimumab or placebo respectively (Table 1). Treatment related adverse events (TRAEs) of any type were similar on each arm; however, there was a higher incidence of serious adverse events and immune mediated adverse events in patients receiving ipilimumab (Table 1). Additionally, more patients receiving ipilimumab discontinued therapy due to a TRAE when compared to patients receiving placebo, $18 \%$ vs. $2 \%$. The dose intensity of chemotherapy was not compromised in patients receiving ipilimumab despite the higher rate of discontinuation due to TRAEs (10).

The median progression-free survival (PFS) by investigator assessment was similar on both arms, 4.6 months for EP plus ipilimumab and 4.4 months for EP alone (Table 1). Similarly, the median OS was not improved with the addition of ipilimumab to EP, median 11 months with ipilimumab $v s$. median 10.9 months with placebo, HR 0.94 (95\% CI, 0.81-1.09), $\mathrm{P}=0.3775$ (Table 1). The 1-year OS was $40 \%$ on each arm. The lack of demonstrated OS benefit was not the result of cross-over as $<1 \%$ of patients on each treatment arm received subsequent immunotherapy (10).

Subgroup analysis did not demonstrate evidence for a particular group benefiting from the addition of ipilimumab. There was no OS difference by whether patients received cisplatin or carboplatin. Similarly, there was no OS difference by presence or absence of baseline central nervous system involvement (10).

\section{IMpower133}

A randomized phase III, double-blind, placebo controlled trial evaluated atezolizumab plus EP vs. EP plus placebo. Carboplatin was the only platinum utilized. Up to 4 cycles of EP were given on each arm. PCI was permitted on either arm, but consolidative thoracic radiotherapy was 
Table 1 Published phase III trials of immune checkpoint inhibitors with first-line platinum plus etoposide in ES-SCLC

\begin{tabular}{|c|c|c|c|c|c|c|c|c|}
\hline Trial & Regimen(s) & $\begin{array}{l}\text { Patient } \\
\text { number }\end{array}$ & ORR & Median PFS & Median OS & TRAEs & $\begin{array}{l}\text { Immune mediated } \\
\text { AEs }\end{array}$ & $\begin{array}{l}\text { Treatment related } \\
\text { SAEs }\end{array}$ \\
\hline \multirow{2}{*}{$\begin{array}{l}\text { Etoposide plus } \\
\text { platinum with or } \\
\text { without } \\
\text { ipilimumab (10) }\end{array}$} & $\begin{array}{l}\text { Ipilimumab plus } \\
\text { etoposide plus } \\
\text { platinum }\end{array}$ & 476 & $62 \%$ & $\begin{array}{l}4.6 \text { months; } \\
\text { HR } 0.85 \\
P=0.0161\end{array}$ & $\begin{array}{l}11.0 \text { months; } \\
\text { HR } 0.94 \\
P=0.3775\end{array}$ & Grade $3-548 \%$ & $\begin{array}{l}\text { Any grade } 57 \% \text {; } \\
\text { grade } 3-520 \%\end{array}$ & Any grade $27 \%$ \\
\hline & $\begin{array}{l}\text { Placebo plus } \\
\text { etoposide plus } \\
\text { platinum }\end{array}$ & 478 & $62 \%$ & 4.4 months & 10.9 months & Grade $3-545 \%$ & $\begin{array}{l}\text { Any grade } 28 \% \\
\text { grade } 3-52 \%\end{array}$ & Any grade $13 \%$ \\
\hline \multirow[t]{2}{*}{$\begin{array}{l}\text { IMpower133 } \\
(7,11)\end{array}$} & $\begin{array}{l}\text { Atezolizumab } \\
\text { plus etoposide } \\
\text { plus platinum }\end{array}$ & 201 & $60 \%$ & $\begin{array}{l}5.2 \text { months; } \\
\text { HR } 0.77 \\
P=0.02\end{array}$ & $\begin{array}{l}12.3 \text { months; } \\
\text { HR } 0.76 \text {, } \\
P=0.0154\end{array}$ & Grade $3-5$ 58.1\% & $\begin{array}{l}\text { Any grade39.9\%; } \\
\text { grade 3-4 10.5\% }\end{array}$ & Any grade $22.7 \%$ \\
\hline & $\begin{array}{l}\text { Placebo plus } \\
\text { etoposide plus } \\
\text { platinum }\end{array}$ & 202 & $64 \%$ & 4.3 months & 10.3 months & Grade $3-5$ 57.6\% & $\begin{array}{l}\text { Any grade } 24.5 \% \text {; } \\
\text { grade } 3-42.5 \%\end{array}$ & Any grade $18.9 \%$ \\
\hline Caspian (8) & $\begin{array}{l}\text { Etoposide plus } \\
\text { platinum }\end{array}$ & 269 & $58 \%$ & 5.4 months & 10.3 months & Grade 3-4 52\% & $\begin{array}{l}\text { Any grade; } 3 \% ; \\
\text { grade } 3-4<1 \%\end{array}$ & Any grade $19 \%$ \\
\hline
\end{tabular}

ORR, objective response rate; PFS, progression-free survival; OS, overall survival; TRAEs, treatment related adverse events; AEs, adverse events; SAEs, serious adverse events; HR, hazard ratio.

not allowed. Atezolizumab or placebo were administered following completion of EP until either progressive disease or other discontinuation criteria were met. Carboplatin was administered at an AUC of 5 on day 1, etoposide at $100 \mathrm{mg} / \mathrm{m}^{2}$ on days 1-3 and atezolizumab at $1200 \mathrm{mg}$ or placebo on day 1 . Each cycle was 21 days. The primary endpoint compared investigator assessed PFS and OS between the two treatments. The median follow-up was 13.9 months (7).

Patients had to have brain metastases treated prior to enrollment and these metastases must have been stable off steroids following local therapy. Approximately $9 \%$ of patients in each treatment group had baseline brain metastases. Patients with autoimmune paraneoplastic syndromes were excluded. Prior chest radiotherapy was permitted if it was part of definitive chemoradiation and diagnosis of ES-SCLC was $>6$ months following completion of curative intent therapy for limited stage disease. There were 22 patients on each arm who received PCI. Adverse events of any type were not significantly increased with the addition of atezolizumab to EP; however, there was a higher incidence of immune mediated adverse events in the group that received atezolizumab (Table 1). The confirmed ORR by investigator assessment was similar between arms, $60 \%$ with atezolizumab plus EP and 64\% with EP (Table 1) (7).

The PFS by investigator assessment was improved with atezolizumab plus EP vs. with EP alone, median 5.2 months vs. 4.3 months, 12 month PFS was $13 \%$ vs. 5\%, HR 0.77 (95\% CI, 0.62-0.96), $\mathrm{P}=0.02$ (Table 1). The median number of atezolizumab doses administered was 7 and the median number of placebo doses administered was 6. The PFS curves were overlapping until about the $50 \%$ mark, suggesting that nearly half of patients do not benefit from the addition of atezolizumab to EP. Median DOR was not different between the arms. However, the percentage of responders maintaining response at 12 months was $15 \%$ for atezolizumab plus EP $v$ s. only $5 \%$ for EP alone (7).

At 2 years of follow-up the OS was improved with atezolizumab added to EP $v s$. with EP alone, median 12.3 vs. 10.3 months, 12 month OS was $52 \%$ vs. $38 \%$, HR 0.76 (95\% CI, 0.54-0.91), $\mathrm{P}=0.0154$ (Table 1) (11). The OS curves on both arms were overlapping until about 7 months, after which point the curves began to separate in favor of atezolizumab plus EP. There was not a significant tail on the OS curve for atezolizumab plus EP and the relative magnitude of OS benefit was not as great as observed with some chemoimmunotherapy combinations in non-small cell 
lung cancer (NSCLC) (7,11-14).

\section{Caspian}

This was a randomized, open label, phase III trial evaluating two chemoimmunotherapy combinations $v s$. EP. Chemoimmunotherapy consisted of EP plus durvalumab with or without tremelimumab. Cisplatin or carboplatin were permitted. The study was powered to compare OS between EP and each of the chemoimmunotherapy arms. However, it was not powered to compare the two different chemoimmunotherapy arms to each other. At the first interim analysis the durvalumab plus EP arm met the predefined threshold for statistical significance, but the durvalumab plus tremelimumab plus EP arm did not. Thus, the first report only described results for the durvalumab plus EP vs. EP comparison (8).

Durvalumab was dosed at $1,500 \mathrm{mg}$ every 3 weeks during chemotherapy and then at the same dose every 4 weeks following completion of chemotherapy. On the experimental arm durvalumab was continued until either disease progression or other discontinuation criteria were met. Chemotherapy was administered for 4 cycles in the chemoimmunotherapy arm and for up to 6 cycles in the chemotherapy alone arm. On the chemotherapy alone arm $63 \%$ of patients completed at least 5 cycles of chemotherapy and $57 \%$ completed 6 cycles of chemotherapy. Cisplatin was dosed at $75-80 \mathrm{mg} / \mathrm{m}^{2}$ on day 1 , carboplatin was dosed at an AUC of 5-6 on day 1 and etoposide was dosed at $80-100 \mathrm{mg} / \mathrm{m}^{2}$ on days $1-3$. Each cycle of chemotherapy or chemoimmunotherapy was administered every 21 days. PCI was permitted in only the chemotherapy alone arm and $8 \%$ of patients on this arm received PCI. Patients having received prior chest radiotherapy were excluded and consolidative chest radiotherapy was not allowed (8).

The confirmed ORR by investigator assessment was improved with durvalumab plus EP vs. with EP alone, 68\% vs. $58 \%$ (Table 1). The median DOR was 5.1 months for both groups; however, the percentage of responders who maintained response at 12 months was greater at $23 \%$ for durvalumab plus EP vs. only 6\% for EP. Adverse events of any type were not significantly increased with the addition of durvalumab to EP. However, immune mediated adverse events of any grade were higher for patients who received durvalumab plus EP (Table 1). The median follow-up was 14 months for censored patients (8).

The investigator assessed median PFS was 5.1 months with durvalumab plus EP and 5.4 months with EP (Table 1).
The PFS curves did not begin to separate until a little past 6 months. The 12-month PFS was improved with durvalumab plus EP vs. EP, $18 \%$ vs. $5 \%$. Overall, there was modest improvement in PFS with the addition of durvalumab to EP, HR 0.78 (95\% CI, 0.65-0.94). The PFS curves suggest that well over half of the patients did not have a PFS benefit from the addition of durvalumab to EP (8).

There was improved OS for durvalumab plus EP vs. EP, median OS was 13 vs. 10.3 months, 18 months OS was 34\% vs. 25\%, HR 0.73 (95\% CI, 0.59-0.91), $\mathrm{P}=0.0047$ (Table 1). Similar to the PFS curves, the OS curves did not begin to separate in favor of durvalumab plus EP until a little past 6 months (8). Unlike chemoimmunotherapy in NSCLC, the OS benefit was more modest and there does not yet appear to be a significant tail on the curve that would suggest that the OS benefit of durvalumab plus EP will greatly improve with longer follow-up (8,12-14).

\section{Subsequent line immune checkpoint inhibition in immunotherapy naïve SCLC}

PD-1 axis inhibition plus EP has become the new standard of care for first-line therapy of ES-SCLC in some parts of the World and will likely become a preferred initial option for newly diagnosed ES-SCLC in other regions. This will render second or subsequent line therapy with PD-1 axis inhibitors and/or CTLA-4 inhibitors as generally ineffective options outside the setting of a clinical trial. Novel combinations utilizing a backbone of PD-1 axis inhibition and/or CTLA4 inhibition may prove beneficial in the future for patients with progressive disease on first-line chemoimmunotherapy. In this section the data on second or subsequent line therapy with PD-1 axis inhibitors and/or CTLA-4 inhibitors in immunotherapy-naïve patients with ES-SCLC is briefly summarized. Each of the regimens discussed below demonstrated prolonged response durations, highlighting the potential of such therapies for some patients.

On single arm studies the PD-1 inhibitors nivolumab and pembrolizumab have both demonstrated efficacy as second or subsequent line therapy of previously treated ESSCLC (Table 2) (15-20). Both of these PD-1 inhibitors are approved in some parts of the World as third line agents. Nivolumab plus the CTLA-4 inhibitor ipilimumab has demonstrated efficacy in second or subsequent line therapy as well, with particularly high response rates and prolonged survival benefit seen in immunotherapy naïve patients with high TMB by whole exome sequencing (17-20) (Table 2). In contrast, PD-L1 staining on tumor cells did not associate 


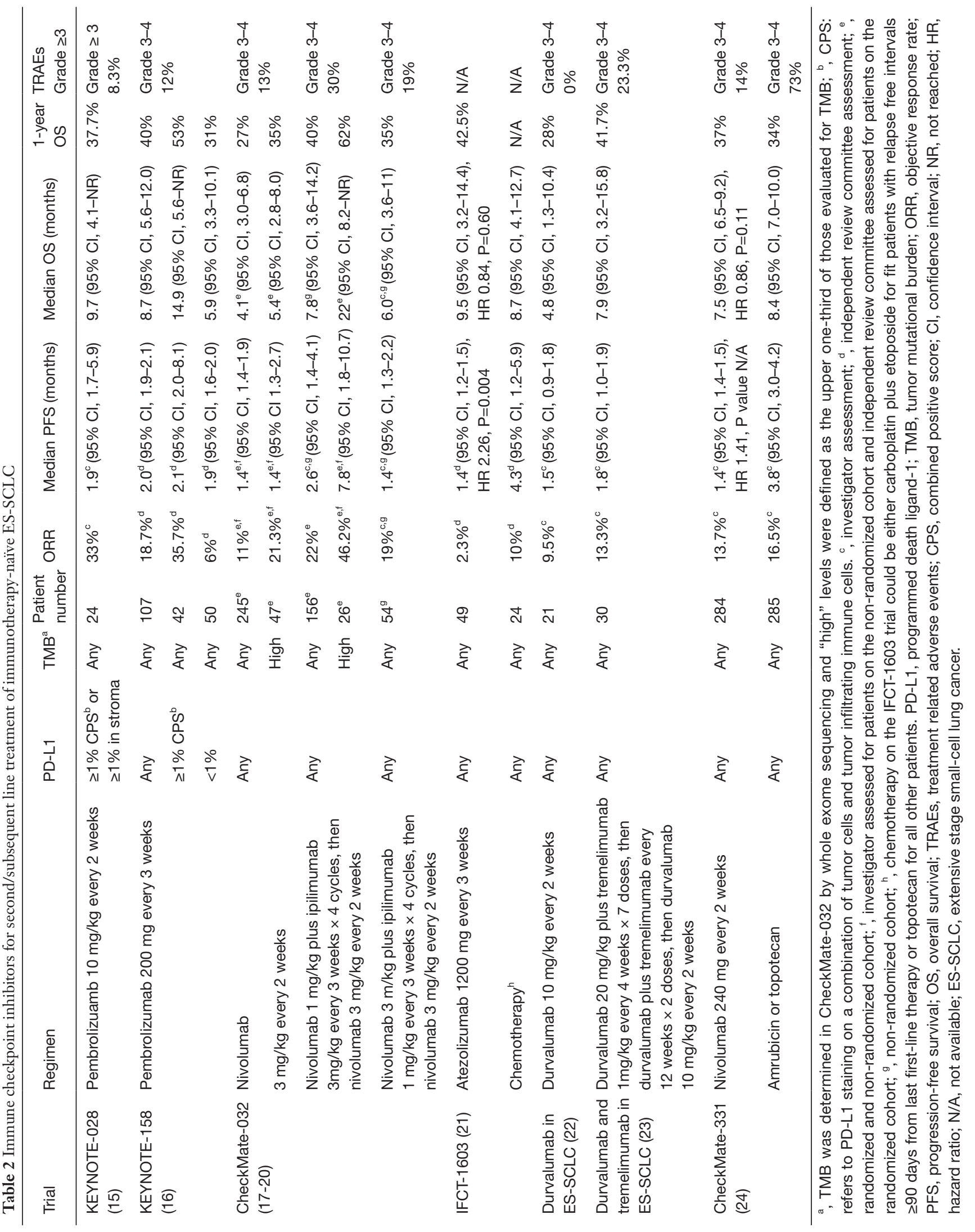


Table 3 Ongoing randomized trials of first-line chemoimmunotherapy in ES-SCLC

\begin{tabular}{|c|c|c|c|c|c|}
\hline Study design & \multicolumn{2}{|c|}{ Regimen(s) } & Primary outcome(s) & $\begin{array}{l}\text { Estimated patient } \\
\text { number }\end{array}$ & $\begin{array}{l}\text { Estimated completion } \\
\text { date }\end{array}$ \\
\hline $\begin{array}{l}\text { Phase III, randomized, } \\
\text { double-blind, } \\
\text { placebo-controlled } \\
\text { (KEYNOTE-604) }\end{array}$ & (I) & $\begin{array}{l}\text { Pembrolizumab plus platinum plus } \\
\text { etoposide; } \\
\text { Placebo plus platinum plus } \\
\text { etoposide }\end{array}$ & PFS and OS & 453 & October $5^{\text {th }}, 2021$ \\
\hline Phase II, randomized & $\begin{array}{l}\text { (I) } \\
\text { (II) }\end{array}$ & $\begin{array}{l}\text { Platinum plus etoposide plus } \\
\text { nivolumab; } \\
\text { Platinum plus etoposide }\end{array}$ & PFS & 150 & June $2^{\text {nd }}, 2020$ \\
\hline $\begin{array}{l}\text { Phase II, randomized, } \\
\text { double-blind, } \\
\text { placebo-controlled }\end{array}$ & (I) & $\begin{array}{l}\text { Carboplatin plus etoposide plus } \\
\text { atezolizumab plus trilaciclib; } \\
\text { Carboplatin plus etoposide plus } \\
\text { atezolizumab plus placebo }\end{array}$ & $\begin{array}{l}\text { Ability to reduce chemotherapy } \\
\text { induced myelosuppression }\end{array}$ & 105 & May $20^{\text {th }}, 2020$ \\
\hline
\end{tabular}

PFS, progression-free survival; OS, overall survival.

with benefit from nivolumab with or without ipilimumab, but a combined PD-L1 score on tumor cells and immune cells did associate with benefit from pembrolizumab (15-20) (Table 2).

Nivolumab was compared against standard of care chemotherapy (topotecan or amrubicin) as second line treatment in patients with progressive disease during or after EP. Nivolumab had significantly worse PFS than either of these chemotherapies. Additionally, OS was not improved with nivolumab (24) (Table 2).

As second line therapy in a randomized phase II trial atezolizumab did not improve outcomes compared to standard of care chemotherapy (topotecan or retreatment with platinum-based doublet). The ORR with atezolizumab was only $2 \%$. The PFS was worse with atezolizumab than with chemotherapy. Additionally, the OS was not improved with atezolizumab (21) (Table 2).

Durvalumab with or without tremelimumab has been evaluated as second or subsequent line therapy for previously treated ES-SCLC. These immunotherapy regimens were not compared head to head against standard of care chemotherapies. The ORRs observed with these immunotherapy regimens were not high. Additionally, in cross-trial comparisons the PFS is worse than with standard of care chemotherapies and there is not a suggestion of significant improvements in OS $(22,23)$ (Table 2).

\section{Ongoing trials}

There are several trials currently evaluating first-line chemoimmunotherapy regimens in newly diagnosed patients with ES-SCLC (Table 3). The KEYNOTE-604 trial is a randomized double-blind, placebo-controlled trial evaluating the addition of the PD-1 inhibitor pembrolizumab to platinum plus etoposide as first-line treatment in 453 patients with ES-SCLC. There has been a press release of initial trial results, but results have yet to be presented at a meeting or in manuscript format. The addition of pembrolizumab to EP significantly improved PFS, HR 0.75 (95\%CI, 0.61-0.91). However, the addition of pembrolizumab to EP failed to meet the prespecified boundary for statistical significance with regards to OS [HR 0.80 (95\% CI, 0.64-0.98)], despite the confidence interval not overlapping one (25). Additionally, an ongoing phase II study will help determine whether nivolumab plus EP may have enhanced efficacy compared to EP as first-line therapy for ES-SCLC. Trilaciclib, a cyclin dependent kinase 4/6 inhibitor, is currently being evaluated in combination with atezolizumab plus EP as a first-line therapy (26). These ongoing trials are highlighted in Table 3. For the 


\section{Unanswered questions regarding chemoimmunotherapy in SCLC}

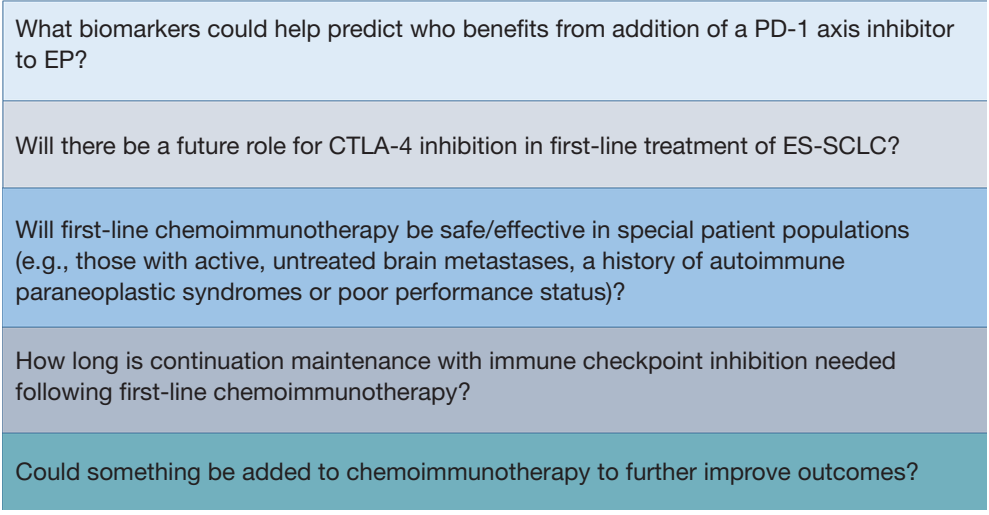

Figure 1 Unanswered questions regarding chemoimmunotherapy in SCLC. SCLC, small-cell lung cancer; PD-1, programmed death-1; EP, etoposide plus platinum; CTLA-4, cytotoxic T-lymphocyte antigen 4; ES-SCLC, extensive-stage small-cell lung cancer.

purposes of this review maintenance trials evaluating switch maintenance with immune checkpoint inhibitors are not discussed as they are not felt most pertinent to the current treatment paradigm where immunotherapy is added to EP from the start of induction treatment.

\section{Discussion}

Multiple questions have arisen following publication of the IMpower133 and Caspian trials. Evaluating the PFS curves on both studies, there were roughly $50 \%$ or more of patients who did not benefit from the addition of a PDL1 inhibitor to EP. Unfortunately, neither trial suggested a biomarker or specific patient characteristic that could help select for who benefits from the addition of a PD-L1 inhibitor to EP $(7,8,11)$ (Figure 1).

On IMpower133, the presence or absence of baseline brain metastases, presence or absence of liver metastases and TMB ( $\geq 10$ mutations per megabase $v s$. $<10$ or $\geq 16$ mutations per megabase $v s .<16$ by a blood based test) did not help select for who benefits from the addition of atezolizumab to EP (7). On Caspian the type of platinum (cisplatin or carboplatin) and presence or absence of baseline brain metastases did not help select for who benefits from the addition of durvalumab to EP. While 39\% of patients on Caspian had baseline liver metastases, survival data for patients with and without baseline liver metastases was not presented (8).

Ipilimumab added to EP did not lead to improved survival outcomes compared to EP alone in first-line treatment of ES-SCLC and at the moment there is no role for CTLA-4 inhibition in first-line therapy. The dose of ipilimumab utilized in conjunction with EP may have contributed to the lack of benefit, as nearly $1 / 5$ of patients had to stop ipilimumab due to TRAEs as opposed to development of progressive disease (10). It is possible a lower dose may have been more beneficial by enabling more patients to stay on therapy longer.

Whether CTLA-4 inhibition plus PD-1 axis inhibition will be beneficial when combined with EP in the first-line setting remains to be determined and ongoing trials will help answer this question (Table 3). The presence of ATrich interactive domain-containing 1A (ARID1A) mutations have been associated with benefit from combined CTLA4 plus PD-L1 inhibition in NSCLC, but not from PDL1 inhibition alone (27). ARID1A mutations are present in $2.5-3.5 \%$ of cases of SCLC (28). Whether mutations in ARID1A associate with benefit from CTLA-4 inhibition plus PD-1 axis inhibition with or without EP in SCLC remains to be determined. DNA polymerase epsilon (POLE) and DNA polymerase delta-1 (POLD1) mutations have been associated with high TMB in other tumor types (29). Presence of these mutations has been suggested to associate with better outcomes in patients receiving immune checkpoint inhibition (30). POLE mutations are present in $2.5-4.5 \%$ of cases of SCLC and POLD1 mutations are present in $1.5-2.5 \%$ of cases of SCLC (28). Whether these mutations help predict benefit from immune checkpoint inhibition plus EP in SCLC is unknown. Kelch-like ECHassociated protein 1 (KEAP1) mutations have been associated with lack of significant improvement from addition of PD-1 inhibition to chemotherapy in NSCLC (31). KEAP1 mutations are present in up to $3.5 \%$ of cases of 
SCLC and whether mutations in KEAP1 may predict lack of benefit from addition of immune checkpoint inhibition to EP in SCLC remains to be determined (28). Further investigation will help determine whether these or other biomarkers may help predict benefit from CTLA-4 inhibition and/or PD-1 axis inhibition in first-line treatment of ES-SCLC (Figure 1).

It is unlikely that CTLA-4 inhibition plus PD-1 axis inhibition will be beneficial for most patients after progression on EP plus a PD-1 axis inhibitor. In melanoma, a disease much more responsive to CTLA4 inhibition, response rates were only about $15 \%$ to ipilimumab following progression on a PD-1 inhibitor (32). Additionally, on CheckMate-032, there were 9 patients who crossed over to nivolumab plus ipilimumab after progression on nivolumab monotherapy and none of these 9 patients had an objective response (18).

Ipilimumab preferentially actives peripheral T-cells, as opposed to intratumoral T-cells. PD-1 axis inhibitors preferentially activate intratumoral T-cells as opposed to peripheral T-cells (33). Ipilimumab did not improve outcomes when added to EP; however, the addition of a PD-L1 inhibitor to EP did improve efficacy $(7,8,10,11)$. Thus, one can hypothesize that activation of intratumoral T-cells may have greater importance than activation of peripheral T-cells for mounting an effective anti-tumor immune response in SCLC and/or that ipilimumab may be more effective if paired with an agent that aids immune cell delivery from the vasculature into the tumor microenvironment (e.g., a vascular endothelial growth factor pathway inhibitor). Whether baseline levels of intratumoral CD4+ and/or CD8+ T-cells could serve as predictors of benefit for addition of PD-1 axis inhibition to $\mathrm{EP}$ in ES-SCLC is unknown.

Some patients with SCLC may have autoimmune paraneoplastic syndromes (6). However, due to safety concerns the phase III trials discussed above did not allow patients with a history of autoimmune paraneoplastic syndromes to enroll $(7,8,10,11)$. Additionally, IMpower133 did not permit patients with asymptomatic untreated brain metastases to enroll; however, the Caspian study did $(7,8,11)$. Unfortunately, the Caspian trial did not present data specific to patients with asymptomatic, untreated brain metastases (8). Also, patients with eastern cooperative oncology group performance status $\geq 2$ were not enrolled on either IMpower133 or Caspian $(7,8,11)$. Whether PD-1 axis inhibition added to EP would be safe and/or effective in the patient populations mentioned above is unknown (Figure 1).
Patients on IMpower133 received up to 4 cycles of EP on the chemotherapy alone arm; however, on Caspian patients received up to 6 cycles of EP on the chemotherapy arm $(7,8,11)$. Whether there is an optimal number of EP cycles to administer in conjunction with PD-1 axis inhibition is unclear. It does appear that 4 cycles could be just as beneficial as 6 cycles, as the median OS was 10.3 months on the control arm of both IMpower133 and Caspian $(7,8,11)$. Whether fewer than 4 cycles of EP can be administered in conjunction with immune checkpoint inhibition without a decrease in efficacy of chemoimmunotherapy is unknown.

On both the IMpower133 and Caspian trials the PFS curves and OS curves did not separate until after 6 months in favor of the chemoimmunotherapy arms $(7,8,11)$. This brings up the possibility that maintenance therapy may be driving some of this benefit. Prior switch maintenance studies involving sequential pembrolizumab or sequential nivolumab plus/minus ipilimumab did not suggest survival improvement following EP for patients with stable disease (SD), PR or CR to induction chemotherapy $(34,35)$. However, both atezolizumab and durvalumab were given as continuation maintenance following induction PD-L1 inhibition plus EP, as opposed to being administered as sequential switch maintenance following EP alone $(7,8,11)$. It is very possible that responses may be longer lasting with immune checkpoint inhibition added from the start of induction chemoimmunotherapy and that continuation maintenance with an immune checkpoint inhibitor may be helping increase the durability of these responses. The only way to definitively determine whether this were true would be a trial where patients were randomized to continuation PD-1 axis inhibition $v s$. observation following completion of induction chemoimmunotherapy. However, such a trial is unlikely to occur because of ethical issues as well as patients not wanting to risk forgoing a generally well tolerated monotherapy treatment.

In both IMpower133 and Caspian the administration of continuation maintenance with $\mathrm{PD}-\mathrm{L} 1$ inhibition occurred until development of progressive disease or other discontinuation criteria were met $(7,8,11)$. However, it is unclear what is the optimal duration of continuation maintenance with atezolizumab or durvalumab for patients with ES-SCLC following induction chemoimmunotherapy (Figure 1). Whether normalization of circulating tumor DNA (ctDNA) by a highly sensitive assay in patients with previously detectable ctDNA levels could help guide who may safely stop PD-L1 inhibition during the maintenance phase is unknown. 


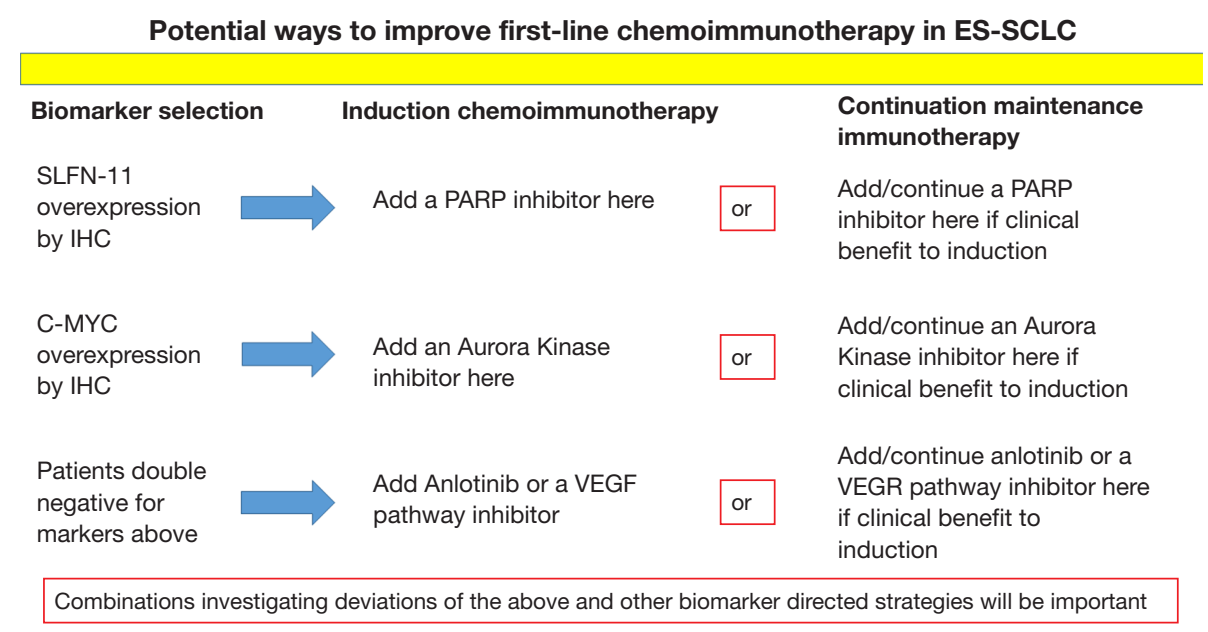

Figure 2 Potential ways to improve first-line chemoimmunotherapy in ES-SCLC. ES-SCLC, extensive-stage small-cell lung cancer; SLFN11, schlafen family member 11; IHC, immunohistochemistry; PARP, poly ADP ribose polymerase; VEGF, vascular endothelial growth factor.

Both the IMpower133 and Caspian trials demonstrated similarly improved OS survival with the addition of PDL1 inhibition to EP. However, the median OS benefit was less than 3 months on both trials and there does not yet appear to be a significant tail on the curves as we have seen with first-line immunotherapy in $\operatorname{NSCLC~(7,8,11-14).~}$ This suggests that perhaps there may be more and/or different immunotherapy resistance mechanisms in SCLC. It is unlikely cross-over masked the OS benefit in either of these trials, as the cross-over to immunotherapy was only $7 \%$ on IMpower133 for the EP alone group and on the Caspian trial it was only $5 \%$ for the EP alone group $(7,8)$. Additionally, PD-1 axis and/or CTLA-4 inhibition have limited survival benefit when administered as second or subsequent line therapy following progression on EP (15-20) (Table 2). Both IMpower133 and Caspian have led to a new standard of care in first-line ES-SCLC; however, there is still a lot of room for improvement.

Recently it has been proposed that SCLC may be divided into four molecular subtypes with distinct gene expression profiles: SCLC-A (high achaete-scute homologue 1), SCLC-N (high neurogenic differentiation factor 1), SCLC-P (high POU class 2 homeobox 3) and SCLC-Y (high yesassociated protein 1). The different immune cell infiltrates and immune response gene signatures in these subtypes has not been reported (36). It could be beneficial to look for associations between these molecular subtypes and efficacy outcomes to chemoimmunotherapy regimens in SCLC.

Oncolytic viruses have been suggested in melanoma to enhance efficacy of immune checkpoint inhibitors $(37,38)$. The Seneca Valley Virus (SVV) is an oncolytic virus that was evaluated as a switch maintenance therapy in ESSCLC patients with at least CR, PR or SD to first-line EP. There was no benefit for SVV as a switch maintenance strategy; however, there was no biomarker selection (39). SVV has selective tropism for SCLC-N, which accounts for an estimated $11 \%$ of cases of SCLC (36). It could be beneficial to investigate whether patients with SCLC-N have improvement in efficacy from addition of SVV to either the start of induction chemoimmunotherapy or from the beginning of continuation maintenance with a PD-1 axis inhibitor.

Overexpression of schlafen family member 11 (SLFN11) by immunohistochemistry has been associated with improved survival from poly ADP ribose polymerase (PARP) inhibition and it may also be a marker for an inflamed tumor microenvironment (40,41). Additionally, PARP inhibitors have been suggested in preclinical models of SCLC to improve efficacy of PD-1/PD-L1 blockade (42). A small phase II study of the PARP inhibitor olaparib plus durvalumab did not demonstrate a good efficacy signal in patients with progressive disease on EP; however, this study did not utilize a biomarker selection strategy (43). It is possible that patients with SLFN11 overexpression by immunohistochemistry may benefit from a PARP inhibitor added to chemoimmunotherapy from either the start of induction or from the start of continuation maintenance (Figure 2).

In a subsequent line therapy trial for patients 
with ES-SCLC the overexpression of c-MYC by immunohistochemistry was associated with significantly better PFS from the aurora kinase A inhibitor alisertib added to paclitaxel (44). Mouse models of breast cancer have suggested that inhibition of aurora kinase A with alisertib may synergize with PD-L1 inhibition through depletion of myeloid derived suppressor cells, depletion of tumor promoting macrophages and an increase in anti-tumor $\mathrm{T}$ lymphocytes within the tumor microenvironment (45). Whether aurora kinase B inhibition would similarly promote anti-tumor immunity is unknown. It is possible that patients with c-MYC overexpression by immunohistochemistry may benefit from the addition of an aurora kinase inhibitor to chemoimmunotherapy either upon starting induction or from the beginning of continuation maintenance (Figure 2).

Vascular endothelial growth factor (VEGF) pathway inhibition has been demonstrated in preclinical and clinical studies to enhance anti-tumor immunity (46-49). Anlotinib is a multi-kinase inhibitor that also inhibits VEGF signaling. Anlotinib has demonstrated efficacy in patients with previously treated ES-SCLC (50). Patients without overexpression of SLFN11 or c-MYC by immunohistochemistry may benefit from the addition of a VEGF pathway inhibitor or anlotinib to chemoimmunotherapy at either the beginning of induction or from the beginning of continuation maintenance (Figure 2).

Adding a fourth agent into the induction phase with chemoimmunotherapy or a second agent to PD-1 axis inhibition at the start of continuation maintenance may be one avenue to improve on first-line chemoimmunotherapy in ES-SCLC. The markers detailed above may help guide the selection of the appropriate agent in a theoretical biomarker selection trial (Figure 2). Such a trial would be one of the initial biomarker investigative strategies in the first-line setting for ES-SCLC. However, there could be challenges enrolling onto such a trial due to small diagnostic tissue specimens and whether such regimens would be safe/ tolerable remains to be determined.

Recent trials combining PD-L1 inhibitors with EP for newly diagnosed patients with ES-SCLC have changed the standard of care first-line therapy for this disease. However, the improvements seen are marginal and far from sufficient. Construction of patient derived xenografts from circulating tumor cells obtained prior to administration of chemoimmunotherapy, during treatment in patients with clinical benefit and at time of development of progressive disease could prove useful in learning about chemoimmunotherapy biomarkers and resistance mechanisms. Further research into biomarkers for SCLC will be essential to advance the field, as will clinical trials investigating novel combinations.

\section{Acknowledgments}

Funding: None.

\section{Footnote}

Provenance and Peer review: This article was commissioned by the editorial office, Fournal of Thoracic Disease for the series "Small Cell Lung Cancer". This article has undergone external peer review.

Conflicts of Interest: The author has completed the ICMJE uniform disclosure form (available at http://dx.doi. org/10.21037/jtd.2020.01.37). The series "Small Cell Lung Cancer" was commissioned by the editorial office without any funding or sponsorship. JMP served as the unpaid Guest Editor for the series and serves as the unpaid editorial board member of Fournal of Thoracic Disease from Oct 2019 to Sep 2021. JMP is the advisory board/ consulting role for AstraZeneca, Gerson Lehrman Group, Hengrui Pharmaceuticals, Novartis and Pfizer, and received honorarium from Genentech and Takeda, Research funding from Pfizer to institution.

Ethical Statement: The author is accountable for all aspects of the work in ensuring that questions related to the accuracy or integrity of any part of the work are appropriately investigated and resolved.

Open Access Statement: This is an Open Access article distributed in accordance with the Creative Commons Attribution-NonCommercial-NoDerivs 4.0 International License (CC BY-NC-ND 4.0), which permits the noncommercial replication and distribution of the article with the strict proviso that no changes or edits are made and the original work is properly cited (including links to both the formal publication through the relevant DOI and the license). See: https://creativecommons.org/licenses/by-nc-nd/4.0/.

\section{References}

1. Hanna N, Bunn PA Jr, Langer C, et al. Randomized phase III trial comparing irinotecan/cisplatin with etoposide/ cisplatin in patients with previously untreated extensive- 
stage disease small-cell lung cancer. J Clin Oncol 2006;24:2038-43.

2. Lara PN Jr, Natale R, Crowley J, et al. Phase III trial of irinotecan/cisplatin compared with etoposide/cisplatin in extensive-stage small-cell lung cancer: clinical and pharmacogenomic results from SWOG S0124. J Clin Oncol 2009;27:2530-5.

3. Reck M, Horn L, Novello S, et al. Phase II Study of Roniciclib in Combination with Cisplatin/Etoposide or Carboplatin/Etoposide as First-Line Therapy in Patients with Extensive-Disease Small Cell Lung Cancer. J Thorac Oncol 2019;14:701-11.

4. Socinski MA, Smit EF, Lorigan P, et al. Phase III study of pemetrexed plus carboplatin compared with etoposide plus carboplatin in chemotherapy-naïve patients with extensive-stage small-cell lung cancer. J Clin Oncol 2009;27:4787-92.

5. Peifer M, Fernández-Cuesta L, Sos ML, et al. Integrative genome analyses identify key somatic driver mutations of small-cell lung cancer. Nat Genet 2012;44:1104-10.

6. Sebastian M, Koschade S, Stratmann JA. SCLC, Paraneoplastic Syndromes, and the Immune System. J Thorac Oncol 2019;14:1878-80.

7. Horn L, Mansfield AS, Szczęsna A, et al. First-Line Atezolizumab plus Chemotherapy in Extensive-Stage Small-Cell Lung Cancer. N Engl J Med 2018;379:2220-9.

8. Paz-Ares L, Dvorkin M, Chen Y, et al. Durvalumab plus platinum-etoposide veruss platinum-etoposide in firstline treatment of extensive-stage small-cell lung cáncer (CASPIAN): a randomised, controlled, open-label, phase 3 trial. Lancet 2019;394:1929-39.

9. Reck M, Bondarenko I, Luft A, et al. Ipilimumab in combination with paclitaxel and carboplatin as first-line therapy in extensive-disease-small-cell lung cancer: results from a randomized, double-blind, multicenter phase 2 trial. Ann Oncol 2013;24:75-83.

10. Reck M, Luft A, Szczesna A, et al. Phase III Randomized Trial of Ipilimumab Plus Etoposide and Platinum Vs. Placebo Plus Etoposide and Platinum in Extensive-Stage Small-Cell Lung Cancer. J Clin Oncol 2016;34:3740-8.

11. Reck M, Liu SV, Mansfield AS, et al. IMpower133: Updated Overall Survival (OS) Analysis of First-line (1L) Atezolizumab (Atezo) + Carboplatin + Etoposide in Extensive-Stage SCLC (ES SCLC). Barcelona, Spain: European Society of Medical Oncology Annual Meeting, 2019.

12. Gandhi L, Rodríguez-Abreu D, Gadgeel S, et al. Pembrolizumab plus Chemotherapy in Metastatic NonSmall-Cell Lung Cancer. N Engl J Med 2018;378:2078-92.
13. Paz-Ares L, Luft A, Vicente D, et al. Pembrolizumab plus Chemotherapy for Squamous Non-Small-Cell Lung Cancer. N Engl J Med 2018;379:2040-51.

14. Socinski MA, Jotte RM, Cappuzzo F, et al. Atezolizumab for First-Line Treatment of Metastatic Non-Squamous NSCLC. N Engl J Med 2018;378:2288-301.

15. Ott PA, Elez E, Hiret S, et al. Pembrolizumab in Patients With Extensive-Stage Small-Cell Lung Cancer: Results From the Phase Ib KEYNOTE-028 Study. J Clin Oncol 2017;35:3823-9.

16. Chung HC, Lopez-Martin JA, Kao SCH, et al. Phase 2 study of pembrolizumab in advanced small-cell lung cancer (SCLC): KEYNOTE-158. J Clin Oncol 2018;36:abstr 8506.

17. Antonia SJ, Callahan MK, Awad MM, et al. Impact of Tumor Mutation Burden on the Efficacy of Nivolumab or Nivolumab + Ipilimumab in Small Cell Lung Cancer: An Exploratory Analysis of CheckMate 032. Yokohama, Japan: IASLC 18th World Conference on Lung Cancer, Oct 1518, 2017: Abstr 11063.

18. Antonia SJ, López-Martin JA, Bendell J, et al. Nivolumab alone and nivolumab plus ipilimumab in recurrent smallcell lung cancer (CheckMate 032): a multicentre, openlabel, phase 1/2 trial. Lancet Oncol 2016;17:883-95.

19. Hellmann MD, Callahan MK, Awad MM, et al. Tumor Mutational Burden and Efficacy of Nivolumab Monotherapy and in Combination with Ipilimumab in Small-Cell Lung Cancer. Cancer Cell 2018;33:853-61.e4.

20. Hellman MD, Ott PA, Zugazagoitia J, et al. Nivolumab (nivo) +/- ipilimumab (ipi) in advanced small-cell lung cancer (SCLC): First report of a randomized expansion cohort from CheckMate 032. J Clin Oncol 2017;35:abstr 8503.

21. Pujol JL, Greillier L, Audigier-Valette C, et al. A Randomized Non-Comparative Phase II Study of AntiProgrammed Cell Death-Ligand 1 Atezolizumab or Chemotherapy as Second-Line Therapy in Patients With Small Cell Lung Cancer: Results From the IFCT-1603 Trial. J Thorac Oncol 2019;14:903-13.

22. Goldman JW, Dowlati A, Antonia SJ, et al. Safety and antitumor activity of durvalumab monotherapy in patients with pretreated extensive disease small-cell lung cancer (ED SCLC). J Clin Oncol 2018;36:abstr 8518.

23. Cho DC, Mahipal A, Dowlati A, et al. Safety and clinical activity of durvalumab in combination with tremelimumab in extensive disease small-cell lung cancer (ED-SCLC). J Clin Oncol 2018;36:abstr 85170.

24. Reck M, Vicente D, Ciuleanu T, et al. Randomized Phase 3 Study of Nivolumab Monotherapy Vs. Chemotherapy 
in Relapsed Small Cell Lung Cancer: Results From CheckMate 331. Geneva, Switzerland: ESMO ImmunoOncology Congress, 2018. Ann Oncol 2018;29:x39-x43.

25. Available online: https://investors.merck.com/news/ press-release-details/2020/Mercks-KEYTRUDApembrolizumab-in-Combination-with-ChemotherapySignificantly-Improved-Progression-Free-SurvivalCompared-to-Chemotherapy-Alone-as-First-LineTreatment-for-Extensive-Stage-Small-Cell-Lung-Cancer/ default.aspx. Accessed 2/22/2020.

26. Available online: http://clinicaltrials.gov. Accessed 12/28/2019.

27. Rizvi N, Cho BC, Reinmuth N, et al. Mutations Associated with Sensitivity or Resistance to Immunotherapy in mNSCLC: Analysis from the MYSTIC Trial. Barcelona, Spain: IASLC World Conference on Lung Cancer, 2019.

28. Available online: https://www.cbioportal.org/. Accessed $12 / 28 / 2019$

29. Briggs S, Tomlinson I. Germline and somatic polymerase $\varepsilon$ and $\delta$ mutations define a new class of hypermutated colorectal and endometrial cancers. J Pathol 2013;230:148-53.

30. Wang F, Zhao Q, Wang YN, et al. Evaluation of POLE and POLD1 Mutations as Biomarkers for Immunotherapy Outcomes Across Multiple Cancer Types. JAMA Oncol 2019;5:1504-6.

31. Skoulidis F, Arbour KC, Hellman MD, et al. Association of STK11/LKB1 genomic alterations with lack of benefit from the addition of pembrolizumab to platinum doublet chemotherapy in non-squamous non-small cell lung cancer. J Clin Oncol 2019;37:abstr 102.

32. Zimmer L, Apuri S, Eroglu Z, et al. Ipilimumab alone or in combination with nivolumab after progression on anti-PD-1 therapy in advanced melanoma. Eur J Cancer 2017;75:47-55.

33. Buchbinder EI, Desai A. CTLA-4 and PD-1 Pathways: Similarities, Differences, and Implications of Their Inhibition. Am J Clin Oncol 2016;39:98-106.

34. Gadgeel SM, Pennell NA, Fidler MJ, et al. Phase II Study of Maintenance Pembrolizumab in Patients with Extensive-Stage Small Cell Lung Cancer (SCLC). J Thorac Oncol 2018;13:1393-9.

35. Owonikoko TK, Kim HR, Govindan R, et al. Nivolumab Plus Ipilimumab, Nivolumab, or Placebo as Maintenance Therapy in Patients With Extensive Disease Small Cell Lung Cancer After First-Line Platinum-Based Chemotherapy: Results From the Double-Blind, Randomized Phase 3 CheckMate 451 Study. Geneva, Switzerland: European Lung Cancer Congress, April 10 to
13, 2019.

36. Rudin CM, Poirier JT, Byers LA, et al. Molecular subtypes of small cell lung cancer: a synthesis of human and mouse model data. Nat Rev Cancer 2019;19:289-97.

37. Puzanov I, Milhem MM, Minor D, et al. Talimogene Laherparepvec in Combination with Iipilimumab in Previously Untreated, Unresectable Stage IIIB-IV Melanoma. J Clin Oncol 2016;34:2619-26.

38. Ribas A, Dummer R, Puzanov I, et al. Oncolytic Virotherapy Promotes Intratumoral T Cell Infiltration and Improves Anti-PD-1 Immunotherapy. Cell 2017;170:110919.e10.

39. Schenk EL, Mandrekar SJ, Dy GK, et al. A Randomized Double-Blind Phase II Study of the Seneca Valley Virus (NTX-010) vs. Placebo for Patients with Extensive-Stage SCLC (ES-SCLC) Who Stable or Responding after at Least Four Cycles of Platinum-Based Chemotherapy: North Central Cancer Treatment Group (Alliance) N0923 Study. J Thorac Oncol 2020;15:110-9.

40. Allison Stewart C, Tong P, Cardnell RJ, et al. Dynamic variations in epithelial-to-mesenchymal transition (EMT), ATM and SLFN11 govern response to PARP inhibitors and cisplatin in small cell lung cancer. Oncotarget 2017;8:28575-87.

41. Pietanza MC, Waqar SN, Krug LM, et al. Randomized, Double-Blind, Phase II Study of Temozolomide in Combination With Either Veliparib or Placebo in Patients with Relapsed-Sensitive or Refractory Small-Cell Lung Cancer. J Clin Oncol 2018;36:2386-94.

42. Sen T, Rodriguez BL, Chen L, et al. Targeting DNA Damage Response Promotes Antitumor Immunity through STING-Mediated T-cell Activation in Small Cell Lung Cancer. Cancer Discov 2019;9:646-61.

43. Thomas A, Vilimas R, Trindade C, et al. Durvalumab in Combination with Olaparib in Patients with Relapsed SCLC: Results from a Phase II Study. J Thorac Oncol 2019;14:1447-57.

44. Owonikoko TF, Nackaerts K, Csoszi T, et al. Randomized Phase 2 Study of the Investigational Aurora A Kinase (AAK) Inhibitor Alisertib (MLN8237) + Paclitaxel vs Placebo + Paclitaxel as Second-Line Therapy for Small Cell Lung Cancer. Vienna, Austria: IASLC 17th World Conference on Lung Cancer, 2016.

45. Yin T, Zhao ZB, Guo J, et al. Aurora A Inhibition Eliminates Myeloid Cell-Mediated Immunosuppression and Enhances the Efficacy of Anti-Pd-L1 therapy in Breast Cancer. Cancer Res 2019;79:3431-44.

46. Cheng AL, Qin S, Ikeda M, et al. IMbrave150: Efficacy 
and Safety Results From a $\mathrm{Ph} 3$ Study Evaluating Atezolizumab (atezo) + Bevacizumab (bev) vs Sorafenib (Sor) as First Treatment (tx) for Patients (pts) With Unresectable Hepatocellular Carcinoma (HCC). Signapore: European Society of Medical Oncology Asia Congress, 2019.

47. Meder L, Schuldt P, Thelen M, et al. Combined VEGF and PD-L1 Blockade Displays Synergistic Treatment Effects in an Autochthonous Mouse Model of Small Cell Lung Cancer. Cancer Res 2018;78:4270-81.

Cite this article as: Pacheco JM. Immunotherapy for extensive stage small cell lung cancer. J Thorac Dis 2020;12(10):6212-6224. doi: $10.21037 /$ jtd.2020.01.37
48. Motzer RJ, Penkov K, Haanen J, et al. Avelumab plus Axitinib vs. Sunitinib for Advanced Renal-Cell Carcinoma. N Engl J Med 2019;380:1103-15.

49. Rini BI, Plimack ER, Stus V, et al. Pembrolizumab plus Axitinib vs. Sunitinib for Advanced Renal-Cell Carcinoma. N Engl J Med 2019;380:1116-27.

50. Cheng Y, Wang Q, Li K, et al. Anlotinib as third-line or further-line treatment in relapsed SCLC: a multicentre, randomized, double-blind phase 2 trial. Toronto, Canada: IASLC World Conference on Lung Cancer, 2018. 